\title{
Concise Commentary: Bloating Makes a Comeback in IBS
}

\author{
Giuseppe Chiarioni $^{1}$
}

Published online: 2 January 2019

(c) Springer Science+Business Media, LLC, part of Springer Nature 2019
Bloating and abdominal distension are bothersome symptoms of ambiguous etiology frequently reported by patients with functional gastrointestinal disorders (FGID) [1]. While noticeable abdominal bloating was originally included in the Manning criteria to diagnose irritable bowel syndrome (IBS), the Rome criteria has shifted the attention to abdominal pain [2]. Nevertheless, the majority (60\%) of IBS patients score bloating as their most bothersome symptom [3]. Moreover, the prevalence of bloating in FGID is illdefined for a number of studies that do not rely on standardized diagnostic criteria $[1,4]$.

In this issue of Digestive Diseases and Sciences, Hod and coworkers significantly increased the relevance of bloating to IBS by conducting a cross-sectional study on 250 IBS patients with and without subjective complaints of bloating, comparing them with 254 healthy controls [5]. IBS was diagnosed according to Rome III criteria; healthy volunteers had no history of GI symptoms. A number of factors were considered including IBS subtype, pain severity, symptom severity, comorbidities, psychological disturbances, and dietary restrictions. The vast majority $(88.3 \%)$ of IBS patients suffered from bloating symptoms dating from the time of their initial diagnosis. Additional evaluation was conducted on 101 IBS-Diarrhea (D) subjects and 39 IBS-Constipation (C) subjects. Bloating complaints was associated with higher rates of constipation and lower rates of diarrhea in IBS, though failing to reach statistical significance. The same held true for higher prevalence rates of bloating symptoms and symptom severity when comparing IBS-C with IBS-D patients. Nonetheless, bloating severity significantly correlated with pain severity, IBS symptom severity, psychological disturbances including anxiety and depression, and the prevalence of fibromyalgia. Dietary restrictions for therapeutic purposes were reported more commonly in IBS

Giuseppe Chiarioni

chiarioni@alice.it

1 Division of Gastroenterology, University of Verona, AOUI Verona, Verona, Italy with bloating than in healthy volunteers and in IBS without bloating.

The strengths of the study were the large sample studied and the standardization of IBS diagnosis. Potential weaknesses were the uneven reported balance of IBS subtypes and the lack of a sizable IBS subgroup with mixed bowel habits (IBS-M) [2]. Still, the study provides sound evidence of the relevance of bloating in terms of assessing IBS symptom severity perception, which in turn mirrors its influence on the psychological wellbeing and comorbidities experienced by IBS patients. Furthermore, bloating universally affects IBS patients.

Given the importance of bloating toward the overall condition of IBS patients, this symptom deserves more intense consideration by the Rome Foundation Committee when formulating the next Rome Criteria.

\section{Compliance with ethical standards}

Conflict of interest Dr Chiarioni is member of the Rome Foundation Anorectal Committee.

\section{References}

1. Iovino $\mathrm{P}$, Bucci $\mathrm{C}$, Tremolaterra $\mathrm{F}$, et al. Bloating and functional gastro-intestinal disorders: where are we and where are we going? World J Gastroenterol. 2014;20:14407-14419.

2. Longstreth GF, Thompson WG, Chey WD, et al. Functional bowel disorders. Gastroenterology. 2006;130:1480-1491.

3. Sach J, Bolus R, Fitzgerald L, et al. Is there a difference between abdominal pain and discomfort in moderate to severe IBS patients? Am J Gastroenterol. 2002;97:3131-3138.

4. Jiang X, Locke GR III, Choung RS, et al. Prevalence of abdominal bloating and visible distension: a population-based study. Gut. 2008;57:756-763.

5. Hod K, Ringel-Kulka T, van Tilburg M, Ringel Y. Bloating in irritable bowel syndrome is associated with symptoms severity, psychological factors and comorbidities. Dig Dis Sci. (Epub ahead of print). https://doi.org/10.1007/s10620-018-5352-5. 